NOUVELle

\section{Le jeûne réduit l'inflammation associée aux maladies inflammatoires chroniques sans altérer la réponse immunitaire aux infections aiguës}

Leslie Adda ${ }^{1 *}$, Sara Abou Melhem ${ }^{2 *}$, Jonathan Pol ${ }^{3-5}$
L'inflammation chronique est favorisée par l'excès d'apport calorique

L'inflammation est un processus de réponse naturel d'un organisme face à une agression. Cependant, lorsque celleci est trop importante ou inappropriée, elle peut être à l'origine de phénomènes pathologiques. De nombreuses voies de régulation peuvent moduler, directement ou indirectement, la réponse inflammatoire. Parmi elles, les voies de régulation du métabolisme cellulaire apparaissent de plus en plus clairement impliquées dans le contrôle du statut inflammatoire de nombreuses cellules immunitaires. Domaine de recherche en plein essor, l'immunométabolisme pourrait permettre de révéler des mécanismes physiopathologiques à l'origine de l'apparition de nombreuses maladies. Avec l'occidentalisation des habitudes alimentaires et la consommation importante d'aliments hautement énergétiques, l'excès d'apport calorique est fréquemment observé dans la population mondiale. II serait responsable de l'établissement d'un état inflammatoire systémique chronique [1], en lien avec le développement de maladies métaboliques à composante inflammatoire, telles que le diabète de type 2 ou I'athérosclérose [2]. L'excès d'apport calorique est également impliqué dans la survenue de maladies inflammatoires et auto-immunes [3]. À l'inverse, il a été montré que le jeûne ou les régimes hypocaloriques ont un effet protecteur vis-à-vis de ces maladies [4,5]. Des études cliniques de jeûne intermittent (inférieur à 24 heures) ont montré une réduction des taux de cytokines proinflammatoires, telles que l'interleukine (IL) $1 \beta$, I'IL-6 ou le TNF- $\alpha$ (tumor necrosis factor alpha) [6]. Cependant, les mécanismes par lesquels une réduction de l'apport calorique module l'inflammation systémique restent encore mal compris.

\section{Le pool de monocytes} pro-inflammatoires circulants diminue lors d'un jeûne court C'est dans ce contexte que Jordan et al. ont étudié l'impact du jeûne sur l'homéostasie des cellules immunitaires, et tout particulièrement des monocytes [7]. Ces cellules circulent sélectivement vers les sites inflammatoires et y jouent un rôle clé dans l'initiation et le maintien de l'inflammation via la sécrétion de cytokines pro-inflammatoires, dont I'IL- $1 \beta$, I'IL-6 et le TNF- $\alpha$. Au niveau tissulaire, les monocytes se différencient en macrophages également producteurs de nombreux médiateurs de l'inflammation [8].

Chez des sujets sains, les auteurs ont observé une diminution des monocytes sanguins suite à un jeûne de 19 heures, leur taux restant toutefois dans les limites physiologiques. Une expérience similaire réalisée chez la souris a montré que suite à un jeûne court de 4 heures (équivalent à un jeûne nocturne chez
${ }^{1}$ Master 2 Immunologie Translationnelle et Biothérapies, Mention BMC,

Sorbonne Université Paris, France.

${ }^{2}$ Master 2 Immunologie Intégrative

et Systémique, Mention BMC,

Sorbonne Université, Paris, France.

${ }^{3}$ Équipe 11 labellisée par la Ligue Nationale

contre le Cancer, Centre de Recherche

des Cordeliers, Inserm U1138, Université

de Paris, Sorbonne Université, Paris, France.

${ }^{4}$ Gustave Roussy Cancer Campus, Villejuif, France.

${ }^{5}$ Metabolomics and Cell Biology Platforms, Gustave Roussy Cancer Campus, Villejuif,

France.

* Co-premiers auteurs

leslie.adda@hotmail.fr

sara.abou_melhem@etu.upmc.fr

l'homme), le taux de monocytes avait également diminué, particulièrement le sous-type de monocytes pro-inflammatoires Ly-6Chigh. En parallèle, ils ont remarqué que ces cellules s'étaient accumulées dans la moelle osseuse des souris à jeun [7].

\section{L'axe AMPK/PPAR $\alpha$ hépatique} régule l'homéostasie des monocytes Par la suite, les auteurs ont démontré que plusieurs effecteurs agissant en cascade étaient à l'origine de ce phénomène (Figure I). Dans un premier temps, en bloquant la glycolyse chez des souris nourries, et donc la production d'énergie par les cellules, ils ont observé une diminution des monocytes à des niveaux similaires à ceux obtenus lors du jeûne, suggérant que les niveaux d'énergie cellulaire influent sur le pool de monocytes circulant. Senseur énergétique cellulaire majeur, la kinase AMPK (pour AMP-activated protein kinase) est activée par une augmentation du rapport AMP/ATP cellulaire, reflet d'un bas niveau d'énergie. Après avoir administré un activateur de I'AMPK à des souris, les auteurs ont observé une réduction significative des monocytes sanguins, témoignant de son implication dans l'inhibition de la libération des monocytes de la moelle osseuse vers la circulation sanguine. Cible de l'AMPK, le 


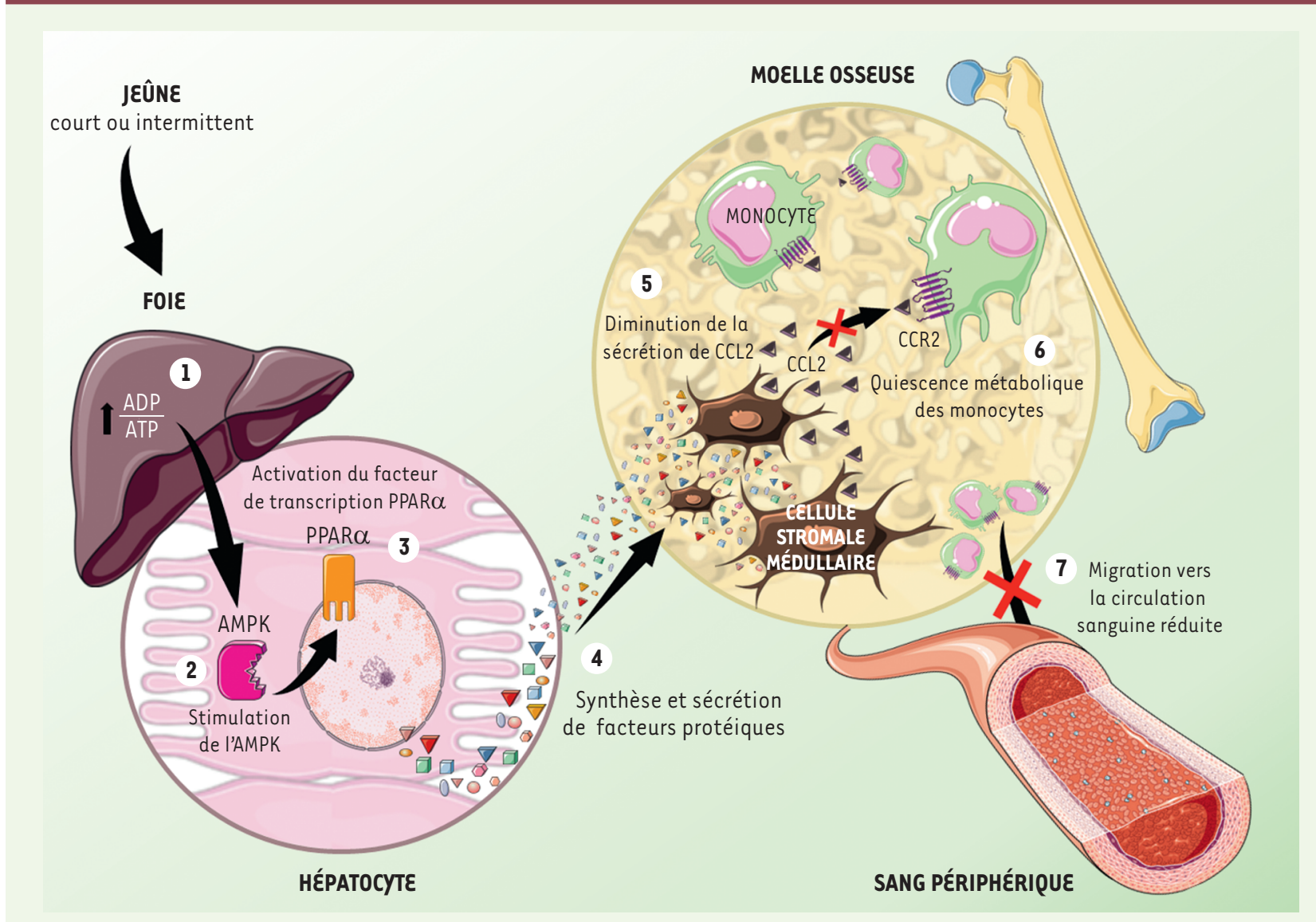

Figure 1. Cascade de régulation du pool de monocytes pro-inflammatoires circulants en condition de jeûne. 1. Le jeûne entraîne l'augmentation du ratio ADP/ATP (ainsi que AMP/ATP) dans les hépatocytes. 2. Un faible niveau d'énergie cellulaire active le senseur énergétique AMPK. 3. L’AMPK stimule le facteur de transcription PPAR $\alpha$. 4. PPAR $\alpha$ module la production de nombreux facteurs protéiques qui régulent la sécrétion médullaire de CCL2 5. La sécrétion de CCL2 par les cellules stromales médullaires est diminuée. 6. En l'absence de liaison de CCL2 à son récepteur CCR2, les monocytes deviennent quiescents. 7. La diapédèse des monocytes est réduite, ils ne sont plus libérés vers la périphérie. AMPK, AMP-activated protein kinase; PPAR $\alpha$, peroxisome proliferator-activated receptor alpha; CCL2, chemokine ligand 2; CCR2, C-C chemokine receptor type 2.

récepteur activé par les proliférateurs de peroxysomes (PPAR $\alpha$ ) est un régulateur de la transcription intervenant notamment dans la réponse adaptative au jeûne [9]. Les auteurs ont montré que la réduction des monocytes circulants induite par le jeûne chez des souris $P P A R \alpha^{/-}$était plus faible que chez les souris sauvages, suggérant que l'activation de PPAR $\alpha$ régule l'homéostasie des monocytes pendant le jeûne. Cependant, le contrôle de la population de monocytes circulants est régi par PPAR $\alpha$ au niveau hépatique et non dans la moelle osseuse. En effet, des souris PPAR $\alpha^{/-}$ irradiées dont la moelle osseuse a été reconstituée par une greffe de cellules de moelle osseuse issues de souris contrôles, ne présentent pas de réduction des monocytes circulants durant un jeûne, contrairement à des souris sauvages irradiées greffées avec des cellules de moelle osseuse PPAR $\alpha^{\prime-}$. En parallèle, après suppression sélective du facteur de transcription PPAR $\alpha$ dans les hépatocytes, les auteurs ont bien observé une altération de la capacité à moduler la libération des monocytes de la moelle osseuse lors du jeûne.

\section{PPAR $\alpha$ favorise la quiescence} métabolique des monocytes en régulant négativement la sécrétion médullaire de CCL2

Sachant que la libération des monocytes de la moelle osseuse vers la circulation sanguine est dépendante de la liaison de la chimiokine CCL2 (chemokine ligand 2) à son récepteur monocytaire CCR2 ( $C-C$ chemokine receptor type 2) [10], les auteurs ont montré que PPAR $\alpha$ agissait sur les monocytes en régulant négativement CCL2. De fait, la modification du sécrétome hépatique dont l'étude a révélé une modification de la sécrétion d'au moins 33 protéines régulatrices de CCL2 en réponse au jeûne. Chez des souris à jeun, le taux de CCL2 circulant est en effet fortement réduit, et ceci même après l'administration de phénformine, un activateur de l'AMPK. De plus, un apport de glucose chez des souris $\mathrm{CCR}^{-/-}$à jeun n'augmente pas le nombre de monocytes sanguins. En 
parallèle, la réduction de CCL2 induite par le jeûne est perdue chez des souris PPAR $\alpha^{/-}$, même en présence de phénformine. Si CCL2 est naturellement produit dans de nombreux tissus (ex. moelle osseuse, foie, rate), l'action de CCL2 sur les monocytes médullaires est dépendante de sa production locale. Le taux de CCL2 détecté dans la moelle osseuse corrèle d'ailleurs avec celui retrouvé dans le sang. De plus, la délétion du gène codant CCL2 dans les hépatocytes ou une splénectomie n'affectent ni le taux sanguin de CCL2 ni le nombre de monocytes circulants.

Enfin, les auteurs ont observé, après un jeûne court, que la modification du profil d'expression de plus de 2700 gènes au sein des monocytes était à l'origine d'un ralentissement de leur métabolisme énergétique, comme en témoigne la réduction conjointe de leur consommation en oxygène et de l'acidification extracellulaire. Les réarrangements du cytosquelette nécessaires à la migration étant l'un des processus cellulaires les plus énergivores [11], les monocytes persistent alors à l'écart de la circulation sanguine et n'exercent ainsi plus leur action pro-inflammatoire systémique [7].

\section{Le jeûne réduit les symptômes}

d'une maladie inflammatoire chronique sans entraver la réponse inflammatoire aiguë anti-infectieuse La restriction calorique ayant été associée à une protection vis-à-vis de maladies inflammatoires chroniques et auto-immunes [4], les auteurs se sont intéressés à l'effet du jeûne intermittent chez des souris atteintes d'encéphalomyélite auto-immune, modèle expérimental de la sclérose en plaques, dans laquelle l'implication des monocytes pro-inflammatoires est connue [12]. Ils ont montré que le jeûne induisait une amélioration des symptômes associée à une réduction du nombre de monocytes pro-inflammatoires infiltrant la moelle épinière. Ces derniers présentaient d'ailleurs une perte de leur signature transcriptomique proinflammatoire, impliquant notamment I'IL-1 $\beta$ et le TNF- $\alpha$. Par ailleurs, les résultats de cette étude mettant en évidence la réduction de la mobilisation périphérique des monocytes proinflammatoires ont amené les auteurs à se demander si le jeûne altérait la mise en place de réactions inflammatoires aiguës, indispensables pour lutter contre une infection par des agents pathogènes. À la différence d'une privation alimentaire prolongée (supérieure à 48 heures), ils ont montré qu'un jeûne court ne nuit pas à la réponse antimicrobienne de souris infectées par la bactérie Listeria monocytogenes, en ce qui concerne le recrutement monocytaire au site infectieux [7].

\section{Conclusion et perspectives thérapeutiques}

L'ensemble de ces résultats démontre la capacité d'un jeûne court ou intermittent à atténuer les effets délétères des monocytes pro-inflammatoires, sans altérer pour autant leur propriété de lutte contre des agents exogènes. Ces données annoncent probablement l'émergence de nouvelles stratégies thérapeutiques pour la prise en charge de maladies métaboliques, auto-immunes ou inflammatoires chroniques. Parmi elles, les agonistes de PPAR $\alpha$, couramment utilisés pour leurs propriétés hypolipémiantes, ont montré une action anti-inflammatoire intéressante dans des modèles animaux de maladies inflammatoires du côlon, de la peau ou du système nerveux [13]. Si les résultats chez l'homme s'avèrent pour l'instant peu probants et les essais cliniques peu nombreux, les SPPARM $\alpha$, agonistes de PPAR $\alpha$ de nouvelle génération, pourraient ouvrir de nouvelles perspectives thérapeutiques, notamment dans le traitement de l'athérosclérose, du diabète de type 2 ou de la stéatohépatite non alcoolique [13]. Par ailleurs, après l'échec en essai clinique d'un anticorps anti-CCL2 dans le traitement de la polyarthrite rhumatoïde, un autre inhibiteur de CCL2 a récemment montré son efficacité dans un essai clinique de phase II dans le traitement de néphropathies diabétiques, dont le développement implique des macrophages pro-inflammatoires au niveau glomérulaire [14]. Étonnament, chez la souris, l'administration de CCL2 à des doses infinitésimales semble être à l'origine de phénomènes régulateurs anti-inflammatoires prometteurs [15]. Enfin, les résultats de cette étude questionnent également la place du jeûne court dans l'arsenal thérapeutique de maladies autoimmunes, inflammatoires ou métaboliques. $\diamond$

Fasting reduces inflammation associated with chronic inflammatory diseases without affecting the immune response to acute infections

\section{LIENS D'INTÉRÊT}

Les auteurs déclarent n'avoir aucun lien d'intérêt concernant les données publiées dans cet article.

\section{RÉFÉRENCES}

1. Lumeng CN, Saltiel AR. Inflammatory links between obesity and metabolic disease. J Clin Invest 2011 ; $121: 2111-7$.

2. Haslam DW, James WPT. Obesity. Lancet 2005 ; 366 : 1197-209.

3. Manzel A, Muller DN, Hafler DA, et al. Role of Western diet in inflammatory autoimmune diseases. Curr Allergy Asthma Rep 2014 ; 14 : 404.

4. Choi IY, Lee C, Longo VD. Nutrition and fasting mimicking diets in the prevention and treatment of autoimmune diseases and immunosenescence. Mol Cell Endocrinol 2017 ; 455 : 4-12.

5. González OA, Tobia C, Ebersole JL, et al. Caloric restriction and chronic inflammatory diseases. Oral Dis 2012 ; 18 : 16-31.

6. Faris MA-IE, Kacimi S, Al-Kurd RA, et al. Intermittent fasting during Ramadan attenuates proinflammatory cytokines and immune cells in healthy subjects. Nutr Res 2012 ; 32 : 947-55.

7. Jordan S, Tung N, Casanova-Acebes M, et al. Dietary intake regulates the circulating inflammatory monocyte pool. Cell 2019; 178 : 1102-14.e17.

8. Yang J, Zhang L, Yu C, et al. Monocyte and macrophage differentiation: circulation inflammatory monocyte as biomarker for inflammatory diseases. Biomark Res $2014 ; 2$ : 1.

9. Régnier M, Polizzi A, Lippi Y, et al. Insights into the role of hepatocyte PPAR $\alpha$ activity in response to fasting. Mol Cell Endocrinol 2018 ; 471 : 75-88.

10. Serbina NV, Shi C, Pamer $\varepsilon$ G. Monocyte-mediated immune defense against murine Listeria monocytogenes infection. Adv Immunol 2012 ; 113 : 119-34. 


\section{RÉFÉRENCES}

11. Daniel JL, Molish IR, Robkin L, et al. Nucleotide exchange between cytosolic ATP and F-actin-bound ADP may be a major energy-utilizing process in unstimulated platelets. Eur J Biochem 1986 ; 156 : 677-84.

12. Kouwenhoven M, Teleshova N, Ozenci V, et al. Monocytes in multiple sclerosis: phenotype and cytokine profile. J Neuroimmunol $2001 ; 112$ : 197-205.

13. Bougarne N, Weyers B, Desmet SJ, et al. Molecular actions of PPAR $\alpha$ in lipid metabolism and inflammation. Endocr Rev 2018 ; 39 : 760-802.

14. Menne J, Eulberg D, Beyer D, et al. C-C motif-ligand 2 inhibition with emapticap pegol (NOX-E36) in type 2 diabetic patients with albuminuria. Nephrol Dial Transplant 2017 ; 32 : 307-15.
15. Kaushansky N, Bakos $\varepsilon$, Becker-Herman S, et al. Circulating picomolar levels of CCL2 downregulate ongoing chronic experimental autoimmune encephalomyelitis by induction of regulatory mechanisms. J Immunol 2019 ; 203 : 1857-66. 